\title{
Pretreatment with intraluminal rapamycin nanoparticle perfusion inhibits neointimal hyperplasia in a rabbit vein graft model
}

This article was published in the following Dove Press journal:

International Journal of Nanomedicine

9 October 2010

Number of times this article has been viewed

\author{
Kai Liu* \\ Guangqing $\mathrm{CaO}^{\text {I* }}$ \\ Xiquan Zhang' \\ Ruifang Liu \\ Weiwei Zou ${ }^{3}$ \\ Shuming $\mathrm{Wu}^{\prime}$ \\ 'Departments of Cardiovascular \\ Surgery, ${ }^{2}$ Anesthesia, Qilu Hospital \\ of Shandong University, Jinan; ${ }^{3}$ The \\ School of Pharmaceutical Science, \\ Shandong University, Jinan, Shandong, \\ People's Republic of China; *Kai Liu \\ and Guangqing Cao contributed \\ equally to this work.
}

Purpose: Poly lactic-co-glycolic acid nanoparticles (PLGA-NP) are widely used as a biodegradable biomaterial in medicine. Rapamycin-eluting stents have been used for prevention of restenosis during surgery. This study investigated the effect of pretreatment with intraluminal perfusion of carbopol-encapsulated rapamycin-loaded PLGA nanoparticles (RAP-PLGA-NP) on neointimal hyperplasia in a rabbit vein graft model.

Methods: A segment of common carotid artery was replaced with a segment of external jugular vein in 60 rabbits which were then separated into four treatment groups, ie, Group 1, in which vein grafts were pretreated with intraluminal RAP-PLGA-NP perfusion, Group 2 in which vein grafts underwent equivalent empty vehicle (PLGA-NP) perfusion, Group 3, in which vein grafts received no treatment, and Group 4, which served as a sham operation group receiving normal vein contrast. On postoperative day 28 , the grafts and normal veins were harvested for histologic examination, flow cytometry analysis, and high-performance liquid chromatography measurement.

Results: Compared with Group 1, the intima of the grafts were thickened, the ratio of intimal area to vessel area increased, and the collagen volume index of the vein grafts increased significantly in Groups 2 and 3. The cell proliferation index in Group $1(21.11 \pm 3.15 \%)$ was much lower than that in Group $2(30.35 \pm 2.69 \%)$ and in Group $3(33.86 \pm 8.72 \%)$. By highperformance liquid chromatography measurement, retention of rapamycin was detected in Group $1(11.2 \pm 0.37 \mu \mathrm{g} / 10 \mathrm{mg}) 28$ days after single drug perfusion.

Conclusion: Pretreatment with intraluminal RAP-PLGA-NP perfusion may inhibit neointimal hyperplasia in vein grafts by penetrating into local tissue and limiting cell proliferation.

Keywords: autograft, stenosis, jugular vein, transplants

\section{Introduction}

Autologous saphenous vein grafting has been a cornerstone in coronary surgery for almost four decades. ${ }^{1}$ However, stenosis or occlusion of the vein graft remains high, occurring in up to $15 \%$ of cases in the first postoperative year, and increasing up to $50 \%$ after 10 years. ${ }^{1,2}$ Stenosis of the graft results from a combination of factors, including the intrinsic quality of the vein, collection and preservation techniques, ${ }^{3}$ hemodynamic alteration after grafting, and inflammation and remodeling of the vein. Updated therapeutic strategies to alleviate restenosis or neointimal hyperplasia correspondingly have included collection and preservation of the vein, pharmacotherapy, gene therapy, radiotherapy, and laser and photodynamic therapy., ${ }^{4,5}$ These treatments have shown inhibitory effects on restenosis in animal trials, but still have some drawbacks.
Department of Cardiovascular Surgery, Qilu Hospital of Shandong University, 107\# Wenhua Xilu, Jinan, Shandong 2500 12, People's Republic of China $\mathrm{Tel}+8653182169427$

Fax +8653 I 86927544

Email shumingwumd@I26.com 
Rapamycin is a novel macrolide antibiotic with marked antiproliferative properties. Both experimental and clinical studies have demonstrated its potency in reducing stenosis in stents and grafts by suppressing neointimal hyperplasia. ${ }^{6-8}$ However, some reports have indicated that inhibition of neointimal formation leads to incomplete neointimal coverage associated with a high rate of thrombus formation after stenting. ${ }^{9-11}$ Subacute thrombosis might be caused by delayed recovery of the intima and a hypersensitivity response to the drug stent. ${ }^{11-13}$ Therefore, continued efforts to improve polymer biocompatibility are warranted.

In recent years, biodegradable nanoparticles and bioadhesive polymer-coated vehicles have attracted considerable interest for the prevention of restenosis in vascular graft disease and hold promise to solve problems produced by local drug delivery. Poly lactide-co-glycolide (PLGA) has been approved by the US Food and Drug Administration (FDA) as a biocompatible and biodegradable polymer for clinical application, and has been used extensively in the manufacture of nanoparticles. ${ }^{14,15}$ Carbopol is a good bioadhesive agent, reported to be a promising stabilizer for fabrication of nanoparticles, ${ }^{16}$ and exhibits good retention both in vitro and in vivo. ${ }^{15,17}$ Local delivery of a drug-loaded bioadhesive agent improves inhibition of restenosis mainly due to the bioadhesive surface, good biocompatibility, easy penetration into the vascular wall, and sustained-release capability. Rapamycin encapsulated with several surface-active agents has demonstrated good antiproliferative efficacy in vascular transplantation when locally applied. ${ }^{18-20}$ However, external application of antiproliferative drugs to the vessel is suspected to affect ambient normal tissues. The ideal route of administration needs to be explored.

Guzman et $\mathrm{al}^{21}$ demonstrated that retention or absorption of drugs loaded into PLGA nanoparticles in the arterial wall could be detected after a short, single intraluminal infusion 14 days earlier in an artery injury model. On the basis of a catheter delivery system, uptake of rapamycin-PLGA nanoparticles in the artery was also shown by Miao et al. ${ }^{22}$ However, little information was given on the effect of local luminal administration of rapamycin nanoparticles in the vein graft. We hypothesized that pretreatment with carbopolencapsulated, rapamycin-loaded PLGA nanoparticles (RAPPLGA-NP) by local intravenous perfusion could play a role in penetrating the vessel from the inner wall without contaminating the tissue outside. In this study, the vein was pretreated with RAP-PLGA-NP ex vivo and then moved into a rabbit vein graft model to test this hypothesis in order to open a new avenue for prevention of restenosis after vein grafting.

\section{Materials and methods}

\section{Animal model}

New Zealand albino rabbits weighing $2.6 \pm 0.3 \mathrm{~kg}$ were obtained from the Laboratory Animal Center of Shandong University. Animals received humane care, and the experiments were performed in accordance with the guidelines of the Animal Care and Use Committee of Shandong University. After intramuscular injection of ketamine $2 \mathrm{mg} / \mathrm{kg}$, stable anesthesia was maintained by continuous infusion of pentobarbital administered through the ear vein at a rate of $200 \mu \mathrm{g} / \mathrm{kg} / \mathrm{min}$. The external jugular vein and common carotid artery on the same side were isolated (about $4 \mathrm{~cm}$ in length). Heparin sodium $125 \mathrm{U} / \mathrm{kg}$ was injected intravenously after the vessels were freed, and a segment of vein about $3 \mathrm{~cm}$ in length was resected. The common carotid artery was clamped proximally and distally to the planned anastomosis with two bulldog clips. Both ends of the prepared external jugular vein were anastomosed end-to-side into the common carotid artery with 7-0 Prolene. Once the anastomosis was completed, the original common carotid artery between the two anastomotic ends (about $3 \mathrm{~cm}$ in length) was ligated and resected. The bulldog clips were removed, and the vein graft filled with arterial blood immediately (Figure 1A). Intravenous penicillin $\mathrm{G}$ procaine ( 0.5 million units) was administered during surgery and daily for three days postoperatively. The rabbits were fed aspirin $2 \mathrm{mg} / \mathrm{kg} /$ day for anticoagulation from the second postoperative day until the end of the study. All the operations were performed by the same two surgeons.

\section{Preparation of RAP-PLGA-NP}

Rapamycin and other chemicals used in this study were provided by Shandong Medical Institution, China, and used without any further purification. Carbopol 940 was purchased from the People's Pharmaceutical Factory of Shanghai, China. RAP-PLGA-NP was prepared by the School of Pharmacology at Shandong University according to previously
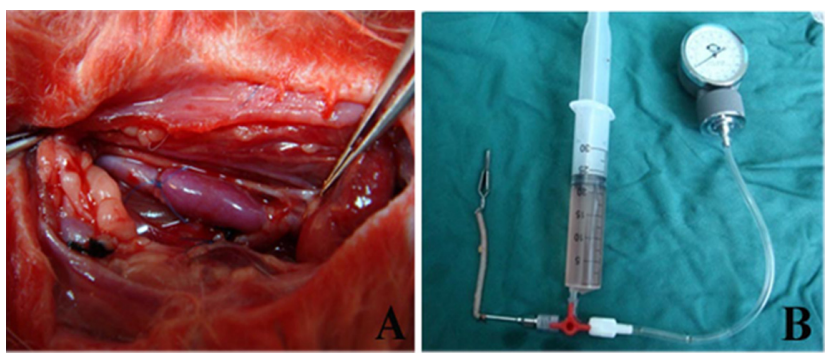

Figure I A) Rabbit model of interposition of external jugular vein into the common carotid artery using an end-to-side technique. B) Self-made drug-perfusion device. 
described methods. ${ }^{23}$ The copolymer was composed of rapamycin and PLGA, and the ratio of rapamycin to PLGA was $0.5: 1(\mathrm{w} / \mathrm{w})$. Finally, the copolymer was encapsulated with carbopol 940, and the concentration of carbopol in the compound was $0.1 \%(\mathrm{w} / \mathrm{v})$.

\section{Definition of groups}

The animals were separated into four groups, ie, Group 1 $(n=15)$ in which the vein grafts were treated with RAPPLGA-NP by local intraluminal perfusion $(500 \mu \mathrm{g} / \mathrm{mL}$, pressure $60 \mathrm{mmHg}$ ) for 180 seconds using a drug infusion device before grafting (Figure 1B), Group $2(n=15)$ in which the vein grafts were pretreated with intraluminal perfusion of an empty vehicle (PLGA-NP) with the same pressure and time as used in Group 1, Group $3(\mathrm{n}=15)$ in which the vein grafts were untreated, and Group $4(n=15)$ in which isolation of the carotid artery and external jugular vein was performed without grafting.

Animals were sacrificed on postoperative day 28 by pentobarbital overdose, after $10 \mathrm{~mL}$ of blood was drawn. Grafts were fixed using 4\% phosphate-buffered formaldehyde via puncture of the left ventricle and the incision of the right atrium. The vein grafts and normal veins were then harvested and fixed in 4\% polyformaldehyde for histopathologic examination. During harvesting, the anastomosis was avoided in the proximal and distal segments of the collected grafts in order to exclude any influence of the anastomosis. Veins were collected without fixation for flow cytometry analysis and high-performance liquid chromatography measurement.

To assess absorption of RAP-PLGA-NP in local vein grafts, a further 40 rabbits were pretreated with high-dose $(500 \mu \mathrm{g} / \mathrm{mL}, \mathrm{n}=20)$ or low-dose $(100 \mu \mathrm{g} / \mathrm{mL}, \mathrm{n}=20)$ intravenous RAP-PLGA-NP perfusion during operation. On postoperative days $7,14,21$, and 28 , five rabbits from each group were sacrificed to test plasma levels and retention of drug in local tissue using high-performance liquid chromatography.

\section{Histopathologic analysis}

The fixed veins were dehydrated using graded alcohols and embedded in paraffin. Slice of $5 \mu \mathrm{m}$ was cut from the proximal, middle, and distal segments of the vein, respectively, and stained with hematoxilyn and eosin and Masson stain. The cross-section of the graft was divided into four quadrants. Three measurements were made in each quadrant, and the median value of all measurements in each crosssection was regarded as the thickness of intima. Because the adventitial area was difficult to distinguish due to adhesion to the surrounding tissue, the intima area (IA) and media area were measured as vessel area (VA). Ratio of intima area to vessel area (IA:VA) was calculated. Collagen volume index (CVI) was represented by means of the ratio of collagen area to the vein wall area on Masson stain slices. The intimal thickness, IA:VA, and CVI of the veins were measured and analyzed using the Pro-Plus 6.0 image system (Media Cybernetics, Inc., Bethseda, MD). All slices were investigated by an experienced pathologist who was blinded to the experimental design. A mean value of measurements was regarded as the representative results of each group.

\section{Flow cytometry assay}

The adventitia of the grafts and normal veins were removed carefully and cut longitudinally. The specimens were washed twice with phosphate-buffered saline and were made into a homogenate. Tissue homogenate were digested by $0.25 \%$ trypsin for 10 minutes and filtered via $75 \mu \mathrm{m}$ mesh to get single cells. The cells were then fixed with precooled ethanol $75 \%$ at $4{ }^{\circ} \mathrm{C}$ overnight. After being washed twice, the cells were loaded with propidium iodide $(65 \mu \mathrm{g} / \mathrm{mL}, \mathrm{pH}$ 7.4; Sigma, St. Louis, MO) containing RNase A (50 $\mu \mathrm{g} / \mathrm{mL}$ in Triscl, pH 7.4; Sigma) for 30 minutes in darkness at room temperature. Each sample containing about $1 \times 10^{6}$ cells was collected. The data were obtained by BD Cellquest software (Becton Dickinson and Company, Franklin Lakes, NJ) and the cell cycle was analyzed by ModFit software (Verity Software House, Topsham, ME). The proliferation index (PI) was represented by the ratio of the $S+G_{2} M$ phase to $G_{0} G_{1}$ phase $\left(P I=\left(S+G_{2} M\right) / G_{0} G_{1}\right)$.

\section{Measurement of rapamycin uptake in vivo and concentration in plasma}

To determine whether rapamycin was retained in the vein graft 28 days after the single short perfusion, the samples were measured using a high-performance liquid chromatography method described previously. ${ }^{23}$ Retention of drug in the samples was expressed as the content of rapamycin $(\mu \mathrm{g})$ per $10 \mathrm{mg}$ tissue. Measurement of one sample was repeated three times and the mean value represented as the ultimate result. To test the rapamycin concentration in plasma, $500 \mu \mathrm{L}$ plasma mixed with $10 \%$ trichloroacetic acid $(200 \mu \mathrm{L})$ and $1 \mathrm{~mL}$ distilled water was centrifuged for 10 minutes at 1500 rpm, and $500 \mu \mathrm{L}$ of the supernatant was analyzed according to the standard curve.

\section{Statistical analysis}

SPSS 10.0 for Windows (SPSS Inc, Chicago, IL) was used for the statistical analysis. Variables were presented as 
mean \pm standard deviation. One-way analysis of variance with a post hoc least significant difference test was used. Comparisons between the two groups were done by $t$-test. $P<0.05$ was considered to be statistically significant.

\section{Results}

Two rabbits, one each in Groups 2 and 3, respectively, were excluded from the study because vein graft thrombus occlusion was detected. One rabbit in the high-dose group died due to an anesthetic accident and one rabbit in the low-dose group was found to have thrombosis on postoperative day 3 . No other complications, such as aneurysmal degeneration or plaque, were observed in any of the animals.

\section{Histologic examination}

Representative photographs of hematoxilyn and eosin and Masson staining are shown in Figures 2 and 3. In Groups 2 and 3, more cells were aggregated at the intima than in Group 1. This might be explained by the antiproliferative effect of RAP-PLGA-NP on neointimal formation. On postoperative day 28 , the intima of grafts were thickened (Figure 4A), and the IA:VA and CVI of the veins were increased significantly (Figure 4B) in Group $2(n=7)$ and

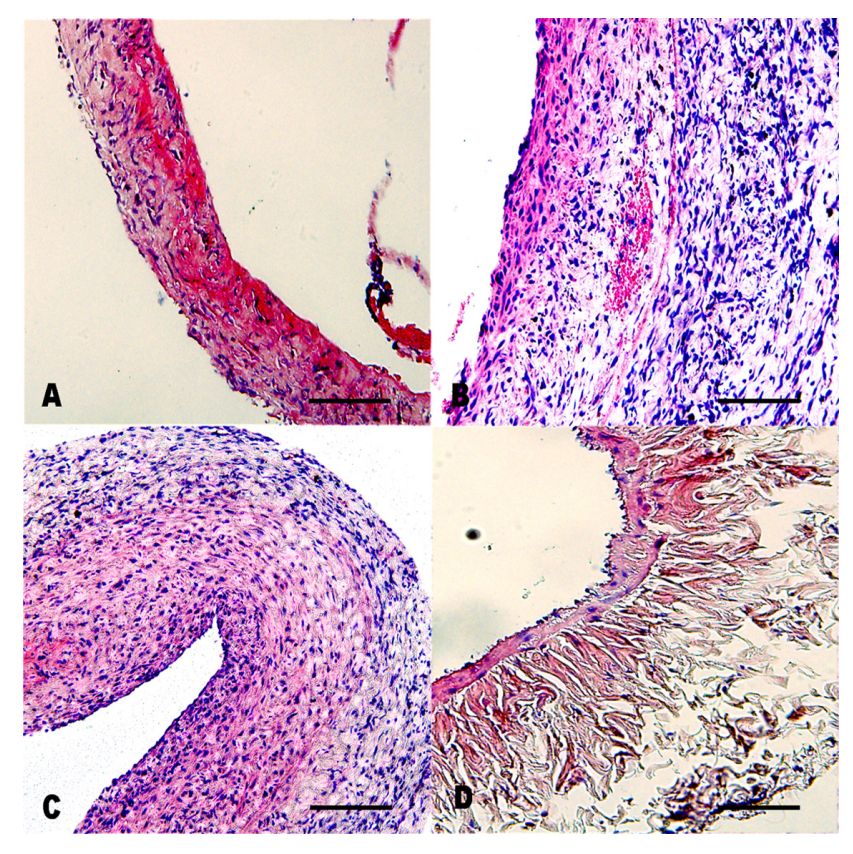

Figure 2 Histopathologic examination of vein grafts and normal vein with hematoxylin and eosin stain 28 days after operation (original magnification 200x). Bar $=100 \mu \mathrm{m}$. A) Group I (rapamycin-loaded PLGA nanoparticle vein graft perfusion), B) Group 2 (empty vehicle), C) Group 3 (vein graft with no treatment), and D) Group 4 (sham operation, normal vein).

Abbreviation: PGLA, poly lactic-co-glycolic acid.

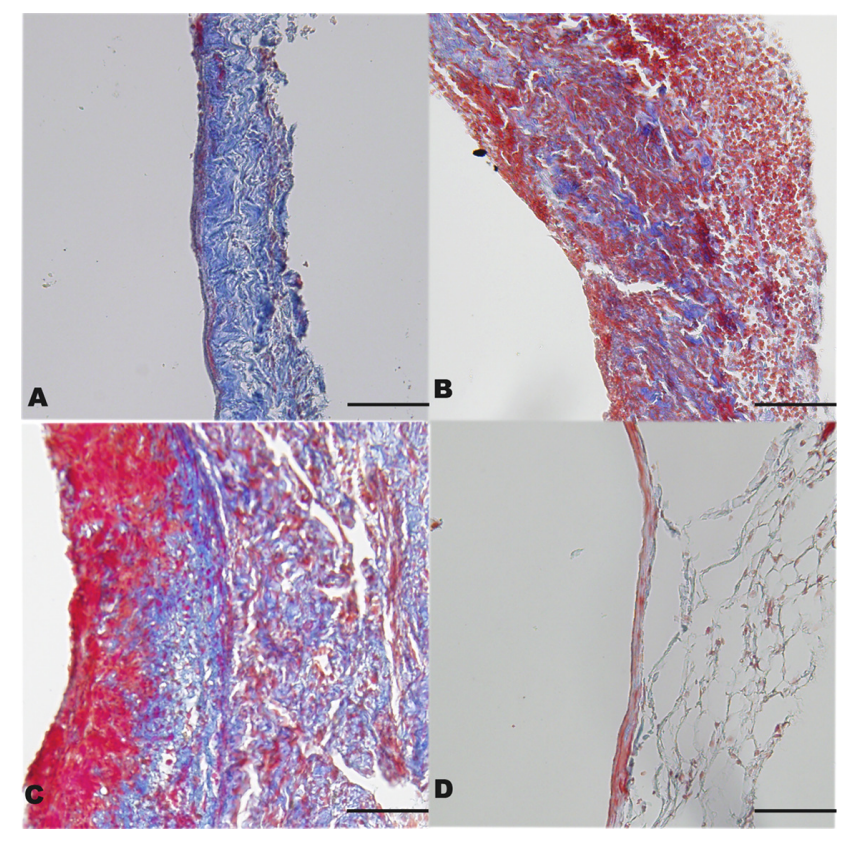

Figure 3 Pathologic examination of vein grafts and normal veins with Masson stain (original magnification 200×). A) Group I (rapamycin-loaded PLGA nanoparticle vein graft perfusion), B) Group 2 (empty vehicle), C) Group 3 (vein graft with no treatment), and D) Group 4 (sham operation, normal vein). In these figures, the collagen was dyed to red. Bar $=100 \mu \mathrm{m}$.

Abbreviation: PGLA, poly lactic-co-glycolic acid.

Group $3(n=7)$ compared with Group $1(n=8)$. Significant differences in intimal thickness and IA:VA were also found between Group 1 and Group $4(n=8)$, but there was a remarkable reduction in graft hyperplasia in Group 1 compared with Groups 2 and 3. There was no significant difference between Group 2 and Group 3 for intimal thickness, IA:VA, and CVI of the grafts. These results indicate that empty vehicle-PLGA-NP had no effect on intimal hyperplasia. Compared with images B and C in Figure 2, we could extrapolate that only PLGA-NP did not induce inflammation in the intima 28 days after the perfusion.

\section{Flow cytometry analysis}

The cell cycles of vein grafts in Groups 1 and 4 were almost stationary at the $\mathrm{G}_{0} \mathrm{G}_{1}$ phase (Figure 5). In Groups 2 and 3, cells in the DNA synthesis phase were significantly increased compared with Groups 1 and 4. The PI in Group $1(n=4)$ was much lower than that in Group $2(\mathrm{n}=4,21.11 \pm 3.15 \%$ versus $30.35 \pm 2.69 \%, P<0.01)$ and Group $3(\mathrm{n}=4$, $21.11 \pm 3.15 \%$ versus $33.86 \pm 8.72 \%, P<0.01)$. Even though the PI of Group 1 was lower than that of Groups 2 and 3, it was higher than that in Group 4 (21.11 $\pm 3.15 \%$ versus $10.99 \pm 2.01 \%, P<0.05)$. There was no significant difference in the PI between Groups 2 and 3 (30.35 $\pm 2.69 \%$ versus $33.86 \pm 8.72 \%, P=0.477$ ). 

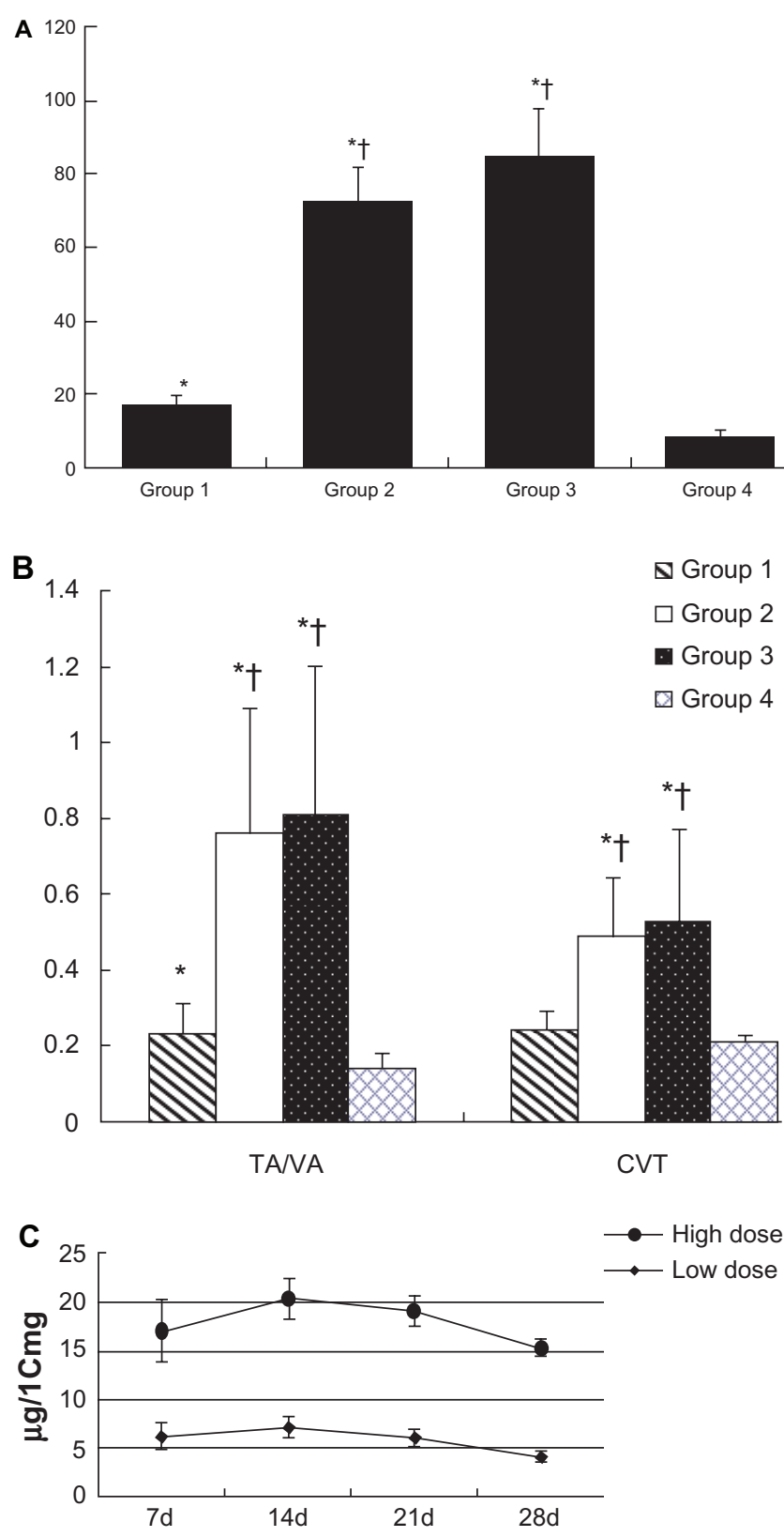

Figure 4 A) Intimal thickness of vein grafts and normal veins on postoperative day 28. $* p<0.05$ compared with Group 4 and ${ }^{t} p<0.05$ compared with Group 1 . B) Ratio of intimal area to vessel area and collagen volume index in the four groups. *P $<0.05$ compared with Group 3 and ${ }^{\dagger} P<0.05$ compared with Group I. Data are expressed as mean \pm standard deviation. C) Retention of rapamycin-loaded PLGA nanoparticles in the local vein graft at different doses. Differences between the two groups existed from seven to 28 days after treatment $(P<0.01)$.

Abbreviations: IA: $\mathrm{VA}$, ratio of intimal area to vessel area; $\mathrm{CVI}$, collagen volume index; PGLA, poly lactic-co-glycolic acid.

\section{Measurement of rapamycin by high- performance liquid chromatography}

Plasma levels of rapamycin were significantly lower than those in vein tissues in both the high-dose group and low-dose group $(P<0.01)$. On postoperative day 28 , rapamycin was detected neither in the high-dose group nor in the low-dose group by high-performance liquid chromatography. At the same postoperative time points, there was no statistically significant difference in plasma concentrations of rapamycin between these two groups (low-dose versus high-dose $1.51 \pm 0.12$ versus $1.69 \pm 0.09 \mathrm{ng} / \mathrm{mL}$, $0.67 \pm 0.07$ versus $0.54 \pm 0.11 \mathrm{ng} / \mathrm{mL}$, and $0.32 \pm 0.05$ versus $0.41 \pm 0.04 \mathrm{ng} / \mathrm{mL}$ on postoperative days 7,14 , and 21, respectively). Retention or uptake of rapamycin in local tissues is shown in Figure 4C. Differences between the low-dose and high-dose groups persisted from days 7 to $28(P<0.01)$. On day 14 after implantation, drug uptake in local tissue peaked and then decreased gradually. Residual rapamycin in the vein graft could be measured in both groups even on postoperative day 28. Liver function was estimated by aspartate aminotransferase and alanine transaminase, and kidney function was represented by blood urea nitrogen and creatinine clearance rate, which were also tested at different time points. No abnormality was observed between the normal animals and the animals treated with RAP-PLGA-NP (data not shown).

Retention of rapamycin in local vein grafts was detected 28 days after the single intraluminal perfusion only in Group 1. The concentration of drug was $11.2 \pm$ $0.37 \mu \mathrm{g} / 10 \mathrm{mg}$ in the local vein graft $(\mathrm{n}=3)$. No rapamycin was detected in the other three groups ( $\mathrm{n}=3$ in each). No rapamycin was detected in plasma after 28 days in any of the groups.

\section{Discussion}

In this study, we investigated the effect of pretreatment with rapamycin nanoparticles by local luminal perfusion on vein graft stenosis. Intravenous perfusion with carbopolencapsulated RAP-PLGA-NP in vivo before grafting could suppress neointimal hyperplasia in a rabbit vein graft model. These results reflect more local penetration of rapamycin nanoparticles into the venous wall to inhibit cell proliferation.

Rapamycin was shown to have a novel pharmacologic effect on prevention of vascular stenosis by blocking cell cycle progression from the G1 phase to the DNA synthesis phase. ${ }^{24}$ This mechanism could be the result of a combination of rapamycin and its specific intracellular receptor, FK506 binding protein 12 (FKBP 12), leading to inactivation of the mammalian target of rapamycin (mTOR), which inhibits the transcription of related genes and DNA synthesis, and subsequently limiting the proliferation and migration of vascular smooth muscle cells. ${ }^{25}$ Both ex vivo and in vivo investigations have confirmed the relationship between mTOR and 

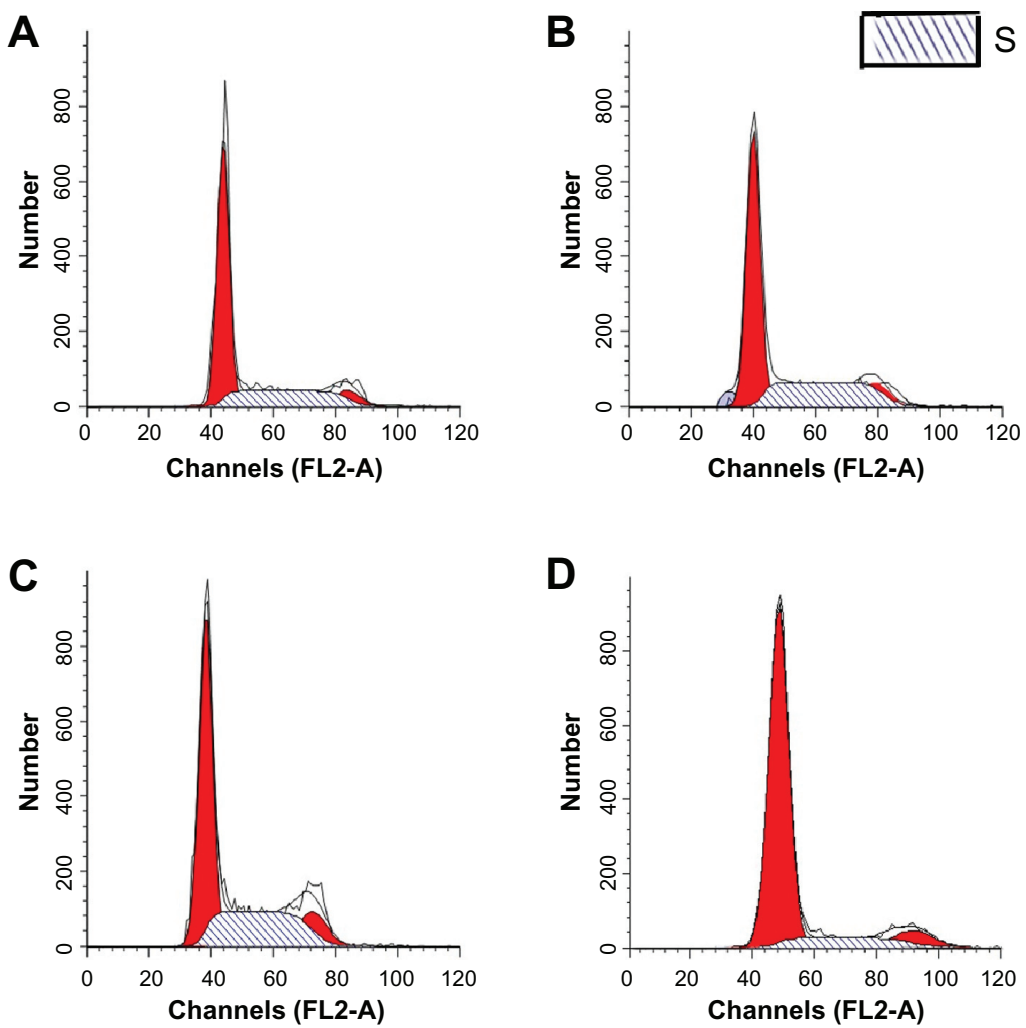

Figure 5 The flow cytometry assay for the proliferation cycles of the cells in the vein grafts and the normal vein. A) Group I (rapamycin-loaded PLGA nanoparticle vein graft perfusion), B) Group 2 (empty vehicle), C) Group 3 (vein graft with no treatment), and D) Group 4 (sham operation, normal vein). S phase, DNA synthesis phase. Abbreviation: PGLA, poly lactic-co-glycolic acid.

rapamycin in neointimal proliferation. ${ }^{26,27}$ During coronary artery bypass surgery, the saphenous vein graft was always preserved in heparinized saline before grafting. Meanwhile, the vein graft could be pretreated with intraluminal RAPPLGA-NP perfusion to prevent future formation of neointimal hyperplasia.

PLGA-NP could prolong drug retention time in tissues and release at a constant velocity to improve drug bioavailability. ${ }^{21}$ Some drugs, including therapeutic antisense, have been loaded into PLGA-NP to prevent or inhibit restenosis in experimental models. ${ }^{28-30}$ In our preliminary study, we found that carbopol 940 was a good carrier, and the release curve of carbopol-encapsulated RAP-PLGA-NP ex vitro fits the Higuchi model. ${ }^{23}$ The present study showed that local intravascular perfusion with RAP-PLGA-NP before grafting had good biocompatibility. After ex vivo treatment, drug retention was also confirmed in local tissue on postoperative day 28. The dose of RAP-PLGA-NP selected was based on preliminary testing. The vein grafts treated with a high dose of drug showed less neointimal hyperplasia than grafts treated with a low dose (data not shown). When the vein grafts were pretreated with rapamycin, the perfusion pressure was limited to $60 \mathrm{mmHg}$ and duration to 180 seconds to allow rapamycin to penetrate into the vein. The infusion device was designed by imitating pressure monitoring during cardiopulmonary bypass. We found the perfusion speed and pressure could be controlled very well during intraluminal perfusion ex vivo. ${ }^{23}$ The materials were easily obtained, and the procedure was simple, without any wasting of time. Furthermore, absorption of rapamycin into the graft was detected without retention of the drug in plasma, as measured by high-performance liquid chromatography in the RAP-PLGA-NP perfusion group on postoperative day 28.

The results of our study showed that intimal thickness, IA:VA, and CVI of the grafts in the RAP-PLGA-NP intraluminal perfusion group were less increased than that in the grafts pretreated with empty vehicle perfusion or without treatment. Thus, we believe that the effects of RAPPLGA-NP on suppression of neointimal hyperplasia were due to direct infiltration into the local target tissue.

Drug retention in the local vein graft measured by highperformance liquid chromatography at different time points suggested that the rapamycin could release consistently in vivo after a short, single perfusion before grafting. The higher retention of drug in local tissue than that in Miao's study might be attributable to the different route of drug 
administration..$^{22}$ In our study, the vein graft was pretreated with RAP-PLGA-NP ex vivo and was then implanted in vivo, whereas in the study by Miao et $\mathrm{al}^{22}$ the drug was delivered by catheter directly to the target tissue under blood flow. No rapamycin was measured in plasma on postoperative day 28. Liver and kidney function were not damaged in the lowdose and high-dose treatment groups. This suggested that slow release of the drug and low blood levels of rapamycin make systemic toxicity unlikely. Moreover, no thrombosis or aneurysm occurred following treatment with RAP-PLGA-NP, which was in accordance with other studies. ${ }^{18-20,31}$ The early thrombus occurring in some of the animals in our study might have been caused by manipulation rather than being an adverse effect of the drug. Previous studies have indicated that the efficacy of rapamycin in stents might not persist for a long time period. ${ }^{31,32}$ It has been suggested that even though retention of the drug could be detected at day 90, intimal proliferation could not be inhibited any further at this time point. ${ }^{32}$ Therefore, the duration of effect of rapamycin in local tissue in vivo after ex vivo treatment needs further investigation. Furthermore, the effect of empty PLGA-NPs in vivo and the most effective dose of RAPPLGA-NP should also be explored, because local toxicity and any adverse effects or complications would influence clinical applicability. ${ }^{11}$

Previous reports have suggested that rapamycin could reduce proliferation of smooth muscle cells by interfering with DNA synthesis, ${ }^{25}$ and therefore PI was selected as an indicator to evaluate the antiproliferative efficacy of rapamycin. In preparation of the cell sample, the adventitia was removed to exclude interference from fibroblasts in the adventitia. This guarantees that the results of flow cytometry analysis which precisely represented cell status in the intima and media. These results indicate that pretreatment with RAP-PLGA-NP could decrease neointimal hyperplasia of vein grafts by limiting cells at the latency stage. In histologic slices, fewer cells were found in the intima in the rapamycin treatment group than in the vehicle or blank groups, which was coincident with the result of flow cytometry analysis. Proliferation was higher in the intraluminal RAP-PLGA-NP perfusion group than that in the normal vein group, but it was much lower than that in the graft without any treatment group. The difference between the intraluminal RAP-PLGA-NP perfusion group and the normal vein might be caused by manipulation and alteration in the hemodynamics of the graft. In fact, proportionate proliferation of intima in vein grafts is desirable in clinical practice after implantation.
The limitation of the current investigation was use of the PI alone as the indicator of cell proliferation, which indirectly reflected neointimal hyperplasia in the grafts. In addition, longer follow-up of the efficacy of RAP-PLGA-NP will be undertaken in future research, and only PLGA-NP will be used in vein grafts in further studies.

\section{Conclusion}

Pretreatment with RAP-PLGA-NP intraluminal perfusion could inhibit intimal hyperplasia in a rabbit model of autologous vein grafting. Local administration of rapamycin nanoparticles mainly limits the cell cycle by inhibiting cell proliferation in tissues in situ. Pretreatment with RAP-PLGA-NP intravascular perfusion would be a potential therapy in vein graft disease.

\section{Acknowledgment}

This study was supported by Grant Number 2008HZ057 from the Natural Science Foundation of Health Department of Shandong Province in China.

\section{Disclosure}

The authors report no conflicts of interest in this work.

\section{References}

1. Shuhaiber JH, Evans AN, Massad MG, Geha AS. Mechanisms and future directions for prevention of vein graft failure in coronary bypass surgery. Eur J CardioThorac Surg. 2002;22(3):387-396.

2. Shah PJ, Gordon I, Fuller J, et al. Factors affecting saphenous vein graft patency: Clinical and angiography study in 1402 symptomatic patients operated on between 1977 and 1999. J Thorac Cardiovasc Surg. 2003;126(6):1972-1977.

3. Rousou LJ, Taylor KB, Lu XG, et al. Saphenous vein conduits harvested by endoscopic technique exhibit structural and functional damage. Ann Thorac Surg. 2009;87(1):62-70.

4. Zilla P, Human P, Wolf M, et al. Constrictive external nitinol meshes inhibit vein graft intimal hyperplasia in nonhuman primates. $J$ Thorac Cardiovasc Surg. 2008;136(3):717-725.

5. Schachner T. Pharmacologic inhibition of vein graft neointimal hyperplasia. J Thorac Cardiovasc Surg. 2006;131(5):1065-1072.

6. Marks AR. Sirolimus for the prevention of in-stent restenosis in a coronary artery. $N$ Engl J Med. 2003;349(14):1307-1309.

7. Schachner T, Oberhuber A, Zou Y, et al. Rapamycin treatment is associated with an increased apoptosis rate in experimental vein grafts. Eur J Cardiothorac Surg. 2005;27(2):302-306.

8. Mehilli J, Byrne RA, Wieczorek A, et al. Randomized trial of three rapamycin-eluting stents with different coating strategies for the reduction of coronary restenosis. Eur Heart J. 2008;29(16):1975-1982.

9. Kotani J, Awata M, Nanto S, et al. Incomplete neointimal coverage of sirolimus-eluting stents. J Am Coll Cardiol. 2006;47(10):2108-2111.

10. Walpoth BH. Adverse effects of local or systemic application of rapamycin for the prevention of neointimal hyperplasia. Ann Thorac Surg. 2005;79(6):2201-2202.

11. Rajathurai T, Rizvi SI, Lin H, Angelini GD, Newby AC, Murphy GJ. Periadventitial rapamycin-eluting microbeads promote vein graft disease in long-term pig vein-into-artery interposition grafts. 2010;3(2): $157-165$.

12. Steffel J, Eberli FR, Lüscher TF, Tanner FC. Drug-eluting stents - what should be improved? Ann Med. 2008;40(4):242-252. 
13. Virmani R, Guagliumi G, Farb A, et al. Localized hypersensitivity and late coronary thrombosis secondary to a sirolimus-eluting stent - should we be cautious? Circulation. 2004;109(6):701-705.

14. Dong Y, Feng SS. Poly(d,1-lactide-co-glycolide)/montmorillonite nanoparticles for oral delivery of anticancer drugs. Biomaterials. 2005;26(30):6068-6076.

15. Kim DH, Martin DC. Sustained release of dexamethasone from hydrophilic matrices using PLGA nanoparticles for neural drug delivery. Biomaterials. 2006;27(15):3031-3037.

16. Vandervoort J, Ludwig A. Biocompatible stabilizers in the preparation of PLGA nanoparticles: A factorial design study. Int J Pharm. 2002; 238(1-2):77-92.

17. Grabovac V, Guggi D, Bernkop-Schnürch A. Comparison of the mucoadhesive properties of various polymers. Adv Drug Deliv Rev. 2005;57(11):1713-1723.

18. Zhang LF, Xiao F, Li J, Wang J, Shi ZH, Li CY. Local applied slowreleasing rapamycin inhibits neointima hyperplasia in vein graft. Zhonghua Yi Xue Za Zhi. 2006;86(24):1706-1709.

19. Schachner T, Zou Y, Oberhuber A, et al. Local application of rapamycin inhibits neointimal hyperplasia in experimental vein grafts. Ann Thorac Surg. 2004;77(5):1580-1505.

20. Kawatsu S, Oda K, Saiki Y, Tabata Y, Tabayashi K. External application of rapamycin-eluting film at anastomotic sites inhibits neointimal hyperplasia in a canine model. Ann Thorac Surg. 2007;84(2):560-567.

21. Guzman LA, Labhasetwar V, Song C, et al. Local intraluminal infusion of biodegradable polymeric nanoparticles. A novel approach for prolonged drug delivery after balloon angioplasty. Circulation. 1996;94(6):1441-1448.

22. Miao LF, Yang J, Huang CL, et al. Rapamycin-loaded poly (lactic-co-glycolic) acid nanoparticles for intrarterial local drug delivery: Preparation, characterization, and in vitro/in vivo release. Zhongguo Yi Xue Ke Xue Yuan Xue Bao. 2008;30(4):491-497.

23. Zou W, Cao G, Xi Y, Zhang N. New approach for local delivery of rapamycin by bioadhesive PLGA-carbopol nanoparticles. Drug Deliv. 2009;16(1):15-23.
24. Bauriedel G, Jabs A, Kraemer S, Nickenig G, Skowasch D. Neointimal expression of rapamycin receptor FK506-binding protein FKBP12: Postinjury animal and human in-stent restenosis tissue characteristics. JVasc Res. 2008;45(2):173-178.

25. Hashemolhosseini S, Nagamine Y, Morley SJ, Desrivières S, Mercep L, Ferrari S. Rapamycin inhibition of the G1 to S transition is mediated by effects on Cyclin Dl mRNA and protein stability. J Biol Chem. 1998;273(23):14424-14429.

26. Semsroth S, Stigler RG, Bernecker OY, et al. Everolimus attenuates neointimal hyperplasia in cultured human saphenous vein grafts. Eur J Cardiothorac Surg. 2009;35(3):515-520.

27. Hu XH, Zhang Q, Yang J, et al. [The expression of mammalian target of rapamycin and its substrates in autogenous vein graft in rats.] Zhonghua Wai Ke Za Zhi. 2006;44(15):1053-1057.

28. Westedt U, Kalinowski M, Wittmar M, et al. Poly (vinylalcohol)graft-poly (lactide-co-glycolide) nanoparticles for local delivery of paclitaxel for restenosis treatment. J Control Release. 2007;19(1): 41-51.

29. Yang J, Zeng Y, Li Y, et al. Intravascular site-specific delivery of a therapeutic antisense for the inhibition of restenosis. Eur J Pharm Sci. 2008;35(5):427-434

30. Feng SS, Zeng W, Teng Lim Y, et al. Vitamin E TPGS-emulsified poly(lactic-co-glycolic acid) nanoparticles for cardiovascular restenosis treatment. Nanomedicine (Lond). 2007;2(3):333-344.

31. Cagiannos C, Abul-Khoudoud OR, DeRijk W, et al. Rapamycin-coated expanded polytetrafluoroethylene bypass grafts exhibit decreased anastomotic neointimal hyperplasia in a porcine model. J Vasc Surg. 2005;42(5):980-988.

32. Carter AJ, Aggarwal M, Kopia GA, et al. Long-term effects of polymerbased, slow-release, sirolimus-eluting stents in a porcine coronary model. Cardiovasc Res. 2004;63(4):617-624.
International Journal of Nanomedicine

\section{Publish your work in this journal}

The International Journal of Nanomedicine is an international, peerreviewed journal focusing on the application of nanotechnology in diagnostics, therapeutics, and drug delivery systems throughout the biomedical field. This journal is indexed on PubMed Central, MedLine, CAS, SciSearch ${ }^{\circledR}$, Current Contents ${ }^{\circledR} /$ Clinical Medicine,

\section{Dovepress}

Journal Citation Reports/Science Edition, EMBase, Scopus and the Elsevier Bibliographic databases. The manuscript management system is completely online and includes a very quick and fair peer-review system, which is all easy to use. Visit http://www.dovepress.com/ testimonials.php to read real quotes from published authors. 